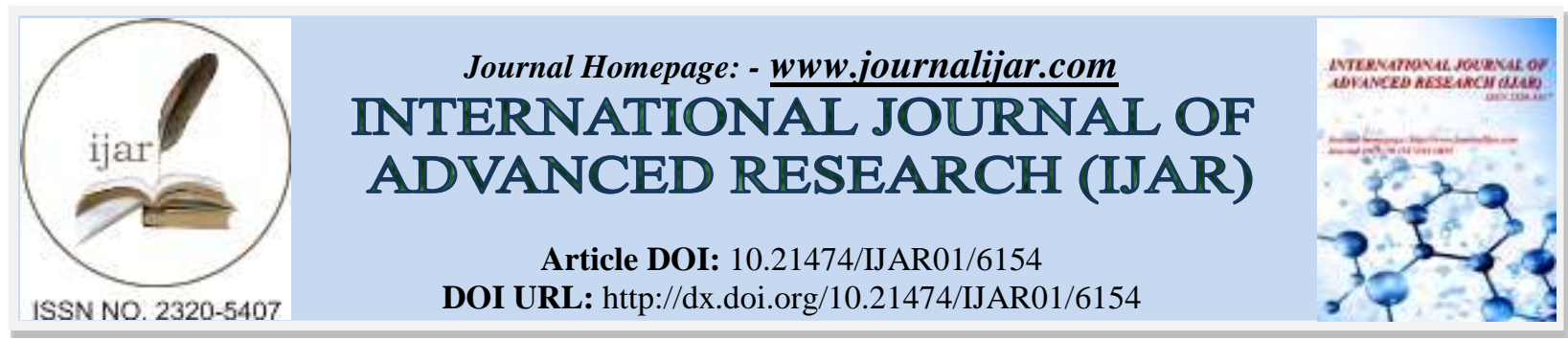

RESEARCH ARTICLE

\title{
MALIGNANT INFANTILE OSTEOPETROSIS (MIOP)-A RARE CASE REPORT
}

1. Associate Professor,2Junior Resident.

\author{
Sunil Kumar Agarwalla ${ }^{1}$ and Nasreen Ali $^{2}$.
}

2. Department of Pediatrics, M.K.C.G Medical College, Berhampur, Ganjam, Odisha- 760004, India.

\section{Manuscript Info}

Manuscript History

Received: 24 October 2017

Final Accepted: 26 November 2017

Published: December 2017

Key words:-

Osteopetrosis, infant, hepatomegaly, splenomegaly, hydrocephalus.

\section{Abstract}

Malignant Infantile Osteopetrosis (MIOP) is a rare genetic disorder due to osteoclast abnormal activity. We report a case of seven month-old female patient, diagnosed as MIOP while investigating the cause of hepatosplenomegaly associated with hydrocephalus.Systemic examination showed hepatosplenomegaly, growth failure and developmental milestones delay. Ophthalmic exam revealed bilateral optic atrophy. Chest radiography detected generalized dense bone.

We report a rare ocular finding of primary optic atrophy in a rare case of malignant variety of osteopetrosis. The patient received supportive treatment. MIOP should be kept in mind as a rare cause of hepatosplenomegaly. Early diagnosis and timely Hematopoietic stem cell transplantation are the only curative approach for an otherwise fatal disease.

Copy Right, IJAR, 2017,. All rights reserved.

\section{Introduction:-}

Osteopetrosis also known as Osteitis Condensans Generalisata, Marble Bone disease, Albers Schonberg disease is a rare disorder of defective osteoclastic resorption of bone, resulting in abnormally dense bones. Two main forms are milder adult onset autosomal dominant form and severe infantile malignant autosomal recessive form. Both forms are due to mutations leading to disturbances of acidification needed for normal osteoclast function due to mutations in the gene ATP6i encoding a subunit of the V-ATPase vacuolar proton pump TC1RG1(OMIM 259700) [1]. Autosomal Recessive MIOP may have the most severe course. Having an incidence of 1:250,000 in general population[2]. Abnormal bone formation and fibrous tissue replace the bone marrow space and finally hematopoesis is decreased. Extramedullar hematopoesis occurs resulting in leukoerythro-blastic anemia and thrombocytopenia. Liver and spleen enlarge progressively. Hemolysis resulting from hypersplenism worsens the anemia and thrombocytopenia. Visual impairment results from bony encroachment on the optic foramina. It is a common initial symptom[3].

\section{Case Report:-}

A seven month-old female infant was referred to the department of pediatric M.K.C.G Medical College and Hospital because of hepatosplenomegaly and thrombocytopenia. Past medical history of the patient revealed that she was hospitalized 1 month back at a local hospital where she was diagnosed as congenital hemolytic anemia but HPLC was normal. The patient was referred to our hospital for further investigation of hepatosplenomegaly and thrombocytopenia. 
She was born after an uncomplicated pregnancy and delivery in the 38th gestational week according to Ballard. Birth weight was 2600 gr. Apgar score was 7/9. No consanguinity in parents.Systemic examination revealed there was nystagmus and blindness, hepatomegaly with the liver noted to be $5 \mathrm{~cm}$, splenomegaly with the spleen noted to be $6 \mathrm{~cm}$. The patient had a bulging anterior fontanel with $4 \times 4 \mathrm{~cm}$ in size. She had pale appearance and fundoscopic examination showed optic atrophy bilaterally.

Laboratory examination yielded the following: hemoglobin $7.5 \mathrm{~g} / \mathrm{dl}$; white blood cell count $30,000 \mathrm{cells} / \mathrm{mm}^{3}$ and platelet count $18.000 / \mathrm{mm}^{3}$ (Figure1). HPLC was normal.Abdominal ultrasonography detected a marked hepatosplenomegaly. Radiologically, X-rays of chest and left upper limb(Figure 2 and 3) show dense homogeneous appearance with characteristic bone-in-bone appearance.

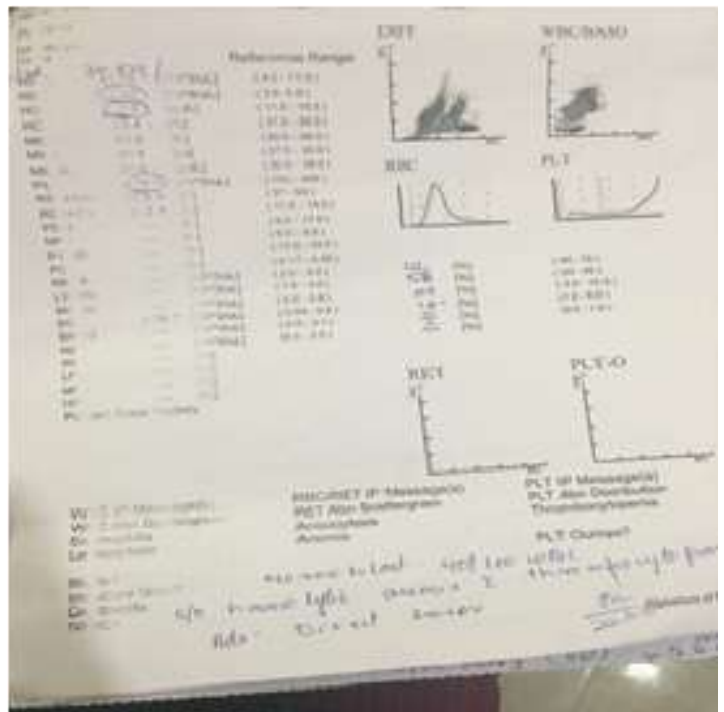

Figure 1:- Showing Pancytopenia

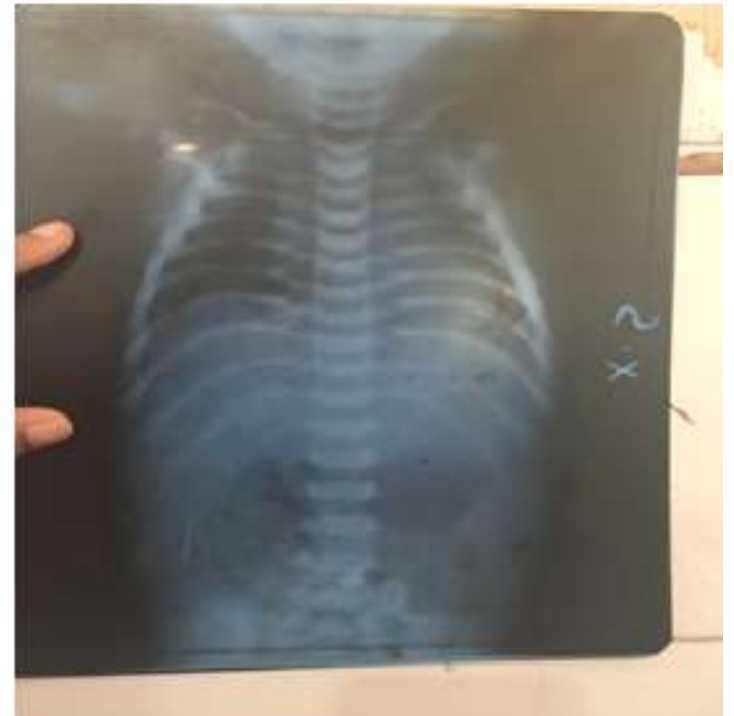

Figure 2 and 3:- showing bone in bone appearance

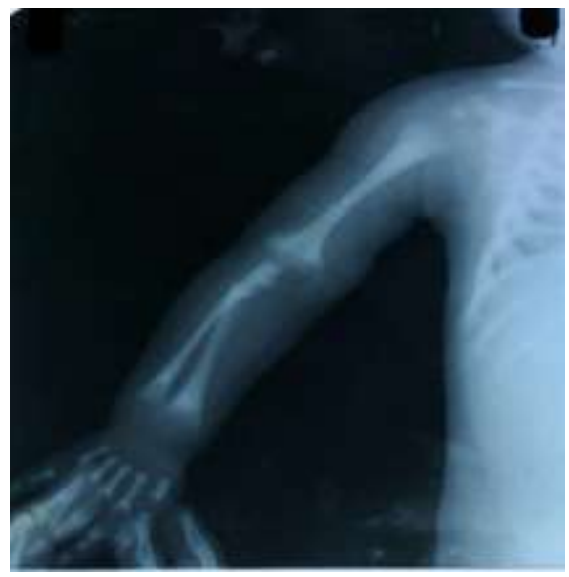

Our initial diagnosis was congenitally acquired infection (TORCH) or a kind of storage disease (like lipid storage or lysosomal diseases) but physical examination and chest radiography confirmed the diagnosis of osteopetrosis. The patient was referred for hematologic stem cell transplantation (SCT).

\section{Discussion:-}

Infantile, or malignant, osteopetrosis is present at birth or develops within the first months of infancy. Our patient had the signs and symptoms from the 3 months of life. Hemolysis resulting from hypersplenism worsens the anemia and thrombocytopenia. Our case was referred for hepatosplenomegaly and peripheral blood smear was significant for leukoerytroblastosis and she needed transfusions for anemia also for thrombocytopenia. In the series reported by Phadke et al[4], optic atrophy was present in three of six cases. In our case optic atrophy was found bilaterally which 
lead to blindness. Radiologic findings showed increased bone density with defective metaphyseal remodeling. The "bone within-bone" appearance is characteristic and diagnostic [5].

Differential diagnoses include congenital disorders (e.g., pseudohypoparathyroidism, pyknodysostosis, and hypoparathyroidism), chemical poisoning (e.g., lead, fluoride, and beryllium), malignancies (myeloproliferative diseases and leukemia),TORCH infections and congenital hemolytic anemia. Based on clinical history and radiographic findings, our case was accepted as the infantile or malignant type, with autosomal recessive inheritance.

Human leucocyte antigen-matched allogeneic haematopoietic stem cell transplantation(to provide the diseased bone with normal osteoclasts) is the only treatment known to significantly alter the course of the disease.[6,7] With younger children the focus should be on obtaining a BMT with the expectation being the preservation of existing sight rather than a reversal of disease process. Children with intact vision must be regarded as urgent priority in regard to BMT waiting lists. Optic canal decompression may be beneficial in some patients but not in others with chorioretinal degeneration. Optic nerve decompression is a hazardous procedure and reports suggest success only in mildly affected older children. Calcitriol (1,25-hydroxyvitamin D3) and Interferon - $1 \mathrm{~b}(1.5 \mu \mathrm{g} / \mathrm{kg}$, three times per week) (macrophage-activating cytokine) have also been used with some benefit.[8] Glucocorticoids may help to stabilize hematopoietic function.6 Symptomatic care such as dental care, transfusions for anaemia, and antibiotic treatment of infections, is important for patients who survive infancy.

Genetic consultation is important. Prenatal diagnosis of osteopetrosis early in pregnancy is an indication for termination of the pregnancy[9]. Our patient was also referred for genetic consultation and mutation analysis but because of financial problems mutation analysis was not performed.

\section{Conclusion:-}

MIOP is a rare disease of infancy. It should be considered in the differential diagnosis of hepatosplenomegaly along with anemia in infancy, having normal HPLC.A radiography can confirm the diagnosis.After the diagnosis the patient must be referred immediately for SCT before neurological impairment and blindness develop.

\section{Reference:-}

1. Frattini A, Orchard PJ, Sobacchi C, et al. Defects in TCIRG1 subunit of the vacuolar proton pump are responsible for a subset of human autosomal recessive osteopetrosis. Nat Genet 2000; 25:343-6.

2. Whyte MP. Osteopetrosis. In: Royce PM, Steinman B, editors. Connective Tissue and its Heritable Disorders: Medical, Genetic, and Molecular Aspects. 2nd ed. New York: Wiley-Liss, Inc.; 2002. pp. 753-70.

3. Venkateshwar V, Voidya A, Roy P, et al. Osteopetrosis. Med J Armed Forces India. 2003;59(4):344-6.

4. Phadke SR, Gupta A, Pahi J, Pandey A, Gautam P, Agarwal SS. Malignant recessive osteopetrosis. Indian Paediatr. 1999;36(1):69-74.

5. Subramaniam A, Singh A, Chavan M, et al. Autosomal recessive osteopetrosis: case report of two siblings. Oral Radiol. 2008;24(2):80-4

6. Gerritsen EJ, Vossen JM, Fasth A, et al. Bone marrow transplantation for autosomal recessive Osteopetrosis : A report from the Working Party on Inborn Errors of the European Bone Marrow Transplantation Group. J Pediatr 1994; 125:896-902.

7. Solh H, Da Cunha AM, Giri N, et al. Bone marrow transplantation for infantile malignant osteopetrosis. J Pediatr Hematol Oncol 1995; 17:350-5.

8. Key LL, Ries WL, Rodriguiz RM, Hatcher HC. Recombinant human interferon gamma therapy for osteopetrosis. J Pediatr 1992; 121:119-24.

9. Besbas N, Draaken M, Ludwig M, et al. A novel CLCN7 mutation resulting in a most severe form of autosomal recessive osteopetrosis. Eur J Pediatr. 2009;168(12):1449-54. 